\title{
Medical Image of the Month: Coccidioidal Pneumatocele Complicated by Pulmonary Hemorrhage
}

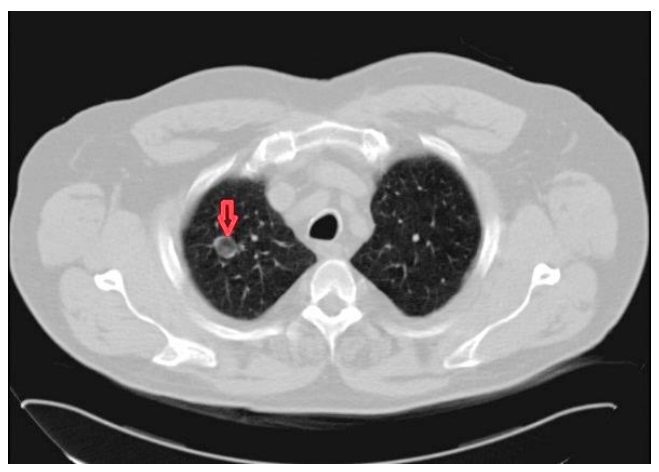

Figure 1. Axial CT of the chest without contrast 12 years prior to this hospitalization demonstrates an irregularly-marginated right upper lobe cyst measuring $1.5 \times 1.6 \mathrm{~cm}$ (red arrow).

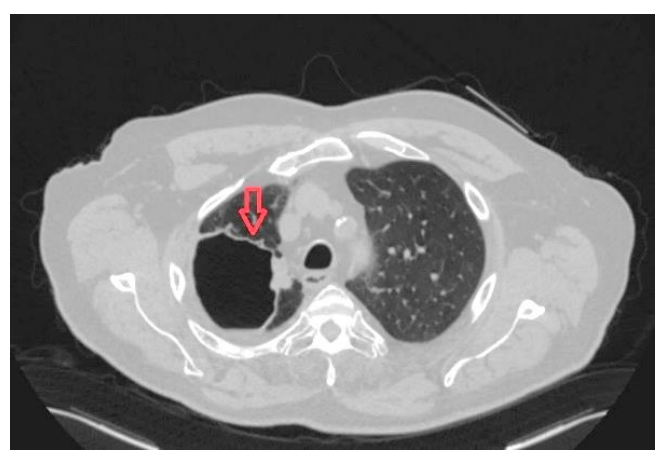

Figure 2. Axial CT of the chest without contrast obtained 4 months prior to this admission demonstrated a cavitary lesion now measuring $6.3 \times 8.2 \mathrm{~cm}$, thin-walled, with small internal air-fluid level and adjacent small pleural effusion without any internal debris (red arrow).

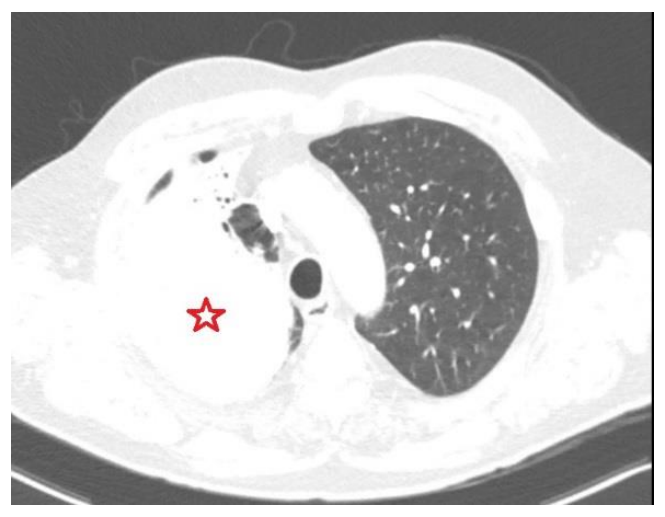

Figure 3. An axial CT angiogram of the chest in lung windows demonstrated a right upper lobe pulmonary cavitary lesion increased in size to $10.5 \mathrm{~cm}$ in largest dimension with almost complete opacification (red star) concerning for a superimposed infection. 
A 77-year-old man with emphysema, hypertension, hypothyroidism, and diabetes mellitus presented with two days of worsening cough that progressed to massive hemoptysis. His hemoptysis included clots the size of golf balls and multiple episodes of frank blood, measuring half a cup each. His symptoms included dyspnea at rest, fatigue, and a 15-20-pound weight loss in three weeks. He denied fevers, night sweats, chest pain, hematemesis, and prior hemoptysis. Additionally, he had a history of coccidioidomycosis complicated by a cavitary lung lesion. Per chart review, 12 years prior to this hospitalization the patient had an irregularly-marginated right upper lobe cyst measuring $1.5 \times 1.6 \mathrm{~cm}$ (Figure 1). A CT scan obtained 4 months prior to admission showed the cavity to be $6.3 \times 8.2 \mathrm{~cm}$ thin-walled and clear of debris (Figure 2) - consistent with a pneumatocele. The patient was referred to thoracic surgery for possible resection at that time but was lost to follow up.

Admission labs showed a decrease in hemoglobin to 13.4 from a baseline of $15.1 \mathrm{~g} / \mathrm{dL}$ and white blood cells of 10,300 cells $/ \mu \mathrm{L}$. Blood cultures were negative. CT angiography now demonstrated an increase in the right upper lobe pulmonary cavitary lesion to 10.5 $\mathrm{cm}$ in largest dimension with almost complete opacification of the lesion - concerning for a superimposed infection. Imaging also showed tree-in-bud nodules in right middle and lower lobes without evidence of a pulmonary embolism (Figure 3). Coccidioidomycosis serologies by EIA showed a non-reactive IgM with reactive IgG. Acid fast bacilli staining of the sputum was negative. Bronchoscopy performed in the hospital showed fresh blood present in the trachea and in the visualized tracheobronchial tree. Active bleeding was noted only from the posterior segment of the right upper lobe. A bronchoalveolar lavage was performed confirming alveolar hemorrhage centered in the right upper lobe. Lidocaine with epinephrine was instilled to stop bleeding. No endobronchial lesion was seen.

The case was evaluated by an interventional radiologist and cardiothoracic surgeon at our institution. They both felt the patient would benefit from transfer to a larger medical center for definitive management of his hemorrhage. He was transferred to a tertiary academic center for a right upper lobectomy, which he tolerated well. Surgical pathology and bronchoscopy cultures ultimately grew coccidioides immitis and the patient was discharge on a treatment course of oral fluconazole.

Pulmonary pneumatoceles are thin-walled, air-filled cystic structures. Most pneumatoceles are encountered in infancy; however, they can appear at any age (1). Pneumatoceles are known sequelae of pneumonia but can also occur due to blunt thoracic injury or as a rare side effect of chemotherapy $(2,3)$. While the mechanism of pneumatocele formation is unclear, several theories have been postulated including check-valve bronchial obstruction and narrowing or from parenchymal necrosis with accompanying focal collections of air within the interstitial tissue (5). Such cases are typically asymptomatic and do not require intervention as they resolve within weeks to months (6). While many pneumatocele resolve on their own without additional intervention, complex pneumatoceles may result in uncontrolled hemorrhage, as portrayed in this case, or infected lesions unresponsive to antibiotics - necessitating surgical intervention (7). Other complications of pneumatoceles are rare and may 
include a tension pneumatocele with cardiorespiratory compromise or pneumothorax (8).

Staphylococcal pneumonia is frequently complicated by pneumatocele development, with pneumatoceles thought to occur in $61 \%$ of cases of staphylococcal pneumonia (9). However, the literature of pneumatocele development following cocci infection is scant. In immunocompetent hosts, infections from coccidiosis are transient, with pulmonary complications (often nodules and self-limited thin-walled cavities) occurring in less than $10 \%$ of patients (10). Complications from coccidiosis infection are usually brief fatigue, dyspnea, cough, and arthritis, with chronic infection or severe complication being rare. Here, we report a case of a gradually enlarging pneumatocele in the setting of cocci infection that eventually eroded into the pulmonary vasculature. The resulting massive hemoptysis was refractory to epinephrine injection and not amenable to catheter embolization. Upper lobectomy was required for definite treatment of the pulmonary hemorrhage.

Sylvester Moses MD, Gregory Gardner MD, Ella Starobinska MD, and Arthur Wolff MD Department of Internal Medicine University of Arizona Tucson, AZ USA

\section{References}

1. Flaherty RA, Keegan JM, Sturtevant HN. Post-pneumonic pulmonary pneumatoceles. Radiology. 1960;74:50-3. [CrossRef] [PubMed]

2. Aissaoui $O$, Alharrar R. Traumatic pulmonary pseudocyst: a rare complication of blunt thoracic injury. Pan Afr Med J. 2019 Apr 11;32:180. [CrossRef] [PubMed]

3. Sangro P, Bilbao I, Fernández-Ros N, Iñarrairaegui M, Zulueta J, Bilbao JI, Sangro $B$. Pneumatocele during sorafenib therapy: first report of an unusual complication. Oncotarget. 2017 Dec 22;9(5):6652-6. [CrossRef] [PubMed]

4. Quigley MJ, Fraser RS. Pulmonary pneumatocele: pathology and pathogenesis. AJR Am J Roentgenol. 1988 Jun;150(6):1275-7. [CrossRef] [PubMed]

5. Zuhdi MK, Spear RM, Worthen HM, Peterson BM. Percutaneous catheter drainage of tension pneumatocele, secondarily infected pneumatocele, and lung abscess in children. Crit Care Med. 1996 Feb;24(2):330-3. [CrossRef] [PubMed]

6. Kaira K, Ishizuka T, Yanagitani N, Sunaga N, Hisada T, Mori M. Pulmonary traumatic pneumatocele and hematoma. Jpn J Radiol. 2009 Feb;27(2):100-2. [CrossRef] [PubMed]

7. Kesieme EB, Kesieme CN, Akpede GO, Okonta KE, Dongo AE, Gbolagade AM, Eluehike SU. Tension pneumatocele due to Enterobacter gergoviae pneumonia: a case report. Case Rep Med. 2012;2012:808630. [CrossRef] [PubMed]

8. Dines DE. Diagnostic significance of pneumatocele of the lung. JAMA. 1968 Jun 24;204(13):1169-72. [CrossRef] [PubMed]

9. Nayeemuddin M, Jankowich MD, Noska A, Gartman EJ. A strange case of coccidioidomycosis: utilization of bronchoscopy to diagnose a chronic cavitary lesion. Am J Resp Crit Care Med. 2018;197:A5427 [Abstract]. 